\title{
Validación del cuestionario acerca de actitudes lingüísticas hacia una lengua extranjera debido al uso de la app Papua
}

Beatriz Peña-Acuña - Universidad de Huelva

$0000-0002-0951-795 X$

Rafael Crismán-Pérez - Universidad de Cádiz

0000-0002-0721-295X

Recepción: 15.06.2021 | Aceptado: 17.07.2021

Correspondencia a través de ORCID: Beatriz Peña-Acuña

0000-0002-0951-795X

Citar: Peña-Acuña, B y Crismán-Pérez, R (2021). Validación del cuestionario acerca de actitudes lingüísticas hacia una lengua extranjera debido al uso de la app Papua. REIDOCREA, 10(28), 1-13. Financiación: Contrato 68/83 con Aprendizaje Inmersivo S.L.

Resumen: La presente investigación consistió en la creación de un instrumento inicial de medición compuesto de 23 preguntas cerradas, el cual medía las actitudes lingüísticas y las actitudes de aprendizaje hacia una lengua extranjera/L2 a partir de herramientas digitales como las aplicaciones (apps). Para la validación del cuestionario, recurrimos a expertos y posteriormente a una muestra de 31 estudiantes con español como primera lengua del Grado de Educación Primaria de la Universidad de Huelva. El cuestionario bilingüe (lengua española e inglés) es una herramienta multidimensional que midió dos dimensiones: la medición de actitudes de aprendizaje/adquisición hacia una lengua extranjera/L2 y la medición de actitudes lingüísticas hacia una lengua extranjera/L2 (CAAAL). Para la elaboración del instrumento se combinó una serie de ítems de acuerdo a las siguientes variables: actitudes hacia el aprendizaje/adquisición de una lengua extranjera/L2; actitudes hacia el aprendizaje/adquisición de las destrezas orales y escritas de una lengua extranjera/L2 y sus posibilidades de interacción y actitudes hacia la motivación del aprendizaje/adquisición de una lengua extranjera/L2. Los resultados demostraron que el instrumento final validado con 13 ítems posee las propiedades psicométricas adecuadas y que resulta válido para medir las dos dimensiones iniciales propuestas.

Palabra clave: Validación cuestionario CAAAL

Validation of a questionnaire about linguistic attitudes towards a second language learning due to Papua app's use

\begin{abstract}
The present research consisted in the creation of an instrument for measuring linguistic attitudes and learning attitudes towards a foreign language / second language from digital tools such as applications (apps). For the elaboration of the questionnaire, we resorted to a sample of 31 students with Spanish as their first language from the Primary Education Degree of the University of Huelva. The bilingual questionnaire (Spanish and English) is a multidimensional tool that measures two dimensions: the measurement of learning attitudes towards a foreign language / second language and the measurement of linguistic attitudes towards a foreign language / second language (CAAAL). For the elaboration of the instrument, a series of items was combined according to the following components: attitudes towards learning; learning/acquisition of a foreign language / second language; attitudes towards the learning / acquisition of oral and written skills of a foreign / second language and their possibilities of interaction and attitudes towards the motivation of learning / acquisition of a foreign / second language. The results showed that the instrument has the appropriate psychometric properties and that it is valid to measure the two proposed dimensions.
\end{abstract}

Keyword: CAAAL questionnaire validation

\section{Introducción}

El objetivo de la presente investigación consiste en la elaboración de un cuestionario validado de análisis de las actitudes y creencias de un grupo de estudiantes universitarios españoles hacia una app para el aprendizaje/adquisición de una lengua extranjera -LE- como segunda lengua -L2-. Para ello recurrimos a una metodología cuantitativa, de modo que llevamos a cabo primeramente el diseño de un cuestionario según un juicio de expertos para la redacción de los ítems. Posteriormente, validamos dicho cuestionario a partir de una muestra de alumnos del Grado de Educación Primaria de $3^{\circ}$ curso de la Universidad de Huelva. 
El interés por elaborar este instrumento proviene de la influencia en la actualidad de las herramientas digitales en el proceso de aprendizaje/adquisición de una LE/L2. El impacto de las herramientas digitales en este proceso es algo ampliamente estudiado en la actualidad tanto desde un punto de vista empírico cuantitativo (González, 2020) como empírico cualitativo (Díaz \& Jansson, 2011), así como teórico (Prensky, 2001; Tapia, 2018).

Dentro del campo de las herramientas digitales, las apps están adquiriendo progresivamente más uso (Echeverría, 1994; García-Aretio, 2016; Lu, 2008). En este punto, hemos puesto nuestra atención en las actitudes que presentan los futuros docentes de enseñanza superior en este tipo de herramientas para la enseñanza de una LE/L2 con el fin de conocer su parecer acerca de la utilidad de estas herramientas en los procesos de enseñanza aprendizaje de LE/L2.

En el caso concreto de la herramienta PAPUA, se trata de un programa para el aprendizaje/adquisición de una LE/L2 a partir del enfoque denominado MALL (Mobile Assisted Learning Language) que incorpora un método inmersivo, basado en la programación de inteligencia artificial para la función del reconocimiento de la voz y la pronunciación del usuario. Este enfoque relaciona la app con el U-learning (Obiquitous Learning, Cope \& Kalantzis, 2009) contribuyendo al desarrollo de la competencia digital (García-Válcarcel \& Martín, 2016). Entre las posibles ventajas de este tipo de aprendizaje con respecto al E-learning destaca la inmediatez de la misma y la diversidad de posibilidades de conexión e interacción con la herramienta mediante app desde varios dispositivos: smartphone y tableta. Además, el profesor puede supervisar los aprendizajes progresivos de los alumnos desde la web de PAPUA (https//: www.papuation.com). Tanto el alumno como el profesor acceden mediante una clave personalizada. Luego se facilita también la acción evaluativa del profesor y autoevaluativa del alumno.

En el programa PAPUA, los contenidos lingüísticos se encuentran organizados en torno a los niveles, A2, B1, B2 y C1, bajo una metodología docente de gamificación. Estos niveles de dificultad son modulables según el alumno desde fácil (A2) a difícil (C1). Además, la modulación del aprendizaje se realiza a través de una serie de apoyos y ayudas por parte de una serie de funciones, por ejemplo, activar o desactivar subtítulos en inglés; activar o desactivar subtítulos en español; nivel de exigencia en el reconocimiento de voz; posibilidad de pedir que te repitan la oración). El proceso de aprendizaje/adquisición se lleva a cabo a partir de pantallas jugables, cada una de ellas de cinco minutos de duración. En cada una de ellas destaca una habilidad por encima de las demás (escribir, leer, hablar, escuchar e interactuar socialmente), de modo que cada pantalla constituye un nuevo escenario virtual de aprendizaje. Durante el juego, el programa evalúa el nivel de comprensión del usuario según las respuestas que este haya ofrecido. La evaluación de la comprensión auditiva y lectora se lleva a cabo mediante pruebas de comprensión implícitas según los contenidos que aparezcan en los diferentes escenarios de aprendizaje. Esto quiere decir que dichas pantallas introducen contenidos de repaso al tiempo que introducen contenidos nuevos para así avanzar en el proceso de aprendizaje/adquisición al tiempo que se afianzan los contenidos previos. También se evalúa el nivel de rendimiento que el alumno haya desarrollado en los escenarios de aprendizaje donde se promueven principalmente las habilidades de hablar, escribir e interactuar según las opciones que haya elegido durante el desenvolvimiento del proceso.

Cada pantalla se desarrolla en torno a una estructura temática, de modo que el programa plantea que en el intervalo de cinco minutos aparezcan los siguientes factores: una habilidad lingüística preponderante, un contenido de acuerdo a un hilo argumental 
o historia, una interacción en una situación cotidiana verosímil y uno o varios contenidos lingüísticos de acuerdo a los niveles de exigencia del MCER. Esta combinación de factores se apoya en tres pilares de la adquisición del lenguaje natural según los modelos de tipo empirista (Bandura, 1976), cognitivo (Chomsky, 1957; 1965) e híbrido (Mayor, 1991). Se trata de los mecanismos de repetición (Skinner, 1957), imitación (Bruner, 1983) y sensación de protección (Cross, 1977).

La herramienta, por tanto, incorpora variables de las principales líneas metodológicas actuales acerca de la adquisición/aprendizaje de una LE/L2 (Gómez, Huertas \& Espejo, 2019). Por una parte, el enfoque cognitivo que tiene en cuenta tanto los procesos mentales internos de gestión de la información como la incorporación de las emociones y las actitudes a los procesos de aprendizaje y adquisición de lenguas. Por otro lado, la corriente tecnológica a partir de la cual se estructuran los procesos virtuales de aprendizaje/adquisición de lenguas desde la focalización en las destrezas comunicativas y sus componentes.

\section{Las actitudes y las actitudes lingüísticas}

En primer lugar, tomamos en consideración el concepto actitud y sus posibilidades de medición. La actitud se define como un estado mental fruto de la experiencia (Allport, G.W., 1935; Azjen, 2001). Este estado mental es lo que permite al individuo relacionarse de una determinada manera con el entorno. La actitud abarca, por tanto, una gran cantidad de campos de la vida, de modo que se define desde una perspectiva estructural de acuerdo a los parámetros actuales de la psicología. Esto se proyecta en esquemas jerarquizados de organización de la información a partir de la dicotomía permanencia/cambio (Parales-Quenza \& Vizcaíno, 2007). Dichos esquemas nos llevan, por tanto, a una caracterización valorativa de las actitudes, puesto que las mismas predisponen al individuo para una determinada respuesta ante un estímulo de la realidad.

Actualmente, la mayoría de los investigadores que han estudiado el concepto actitud se han agrupado en torno a dos posturas fundamentales: el análisis de las actitudes desde un punto de vista conductista y, de manera complementaria, el análisis de las mismas desde un enfoque mentalista (Izquierdo, 2011). No obstante, conforme el estudio de la cuestión ha avanzado, ambas posturas han tendido a encontrarse debido, entre otros motivos, a las influencias complementarias de la psicología social y la psicología cognitiva respectivamente (Eagly \& Chaiken, 1993; 1995; 1998).

En la actualidad, el enfoque predominantemente mentalista caracteriza la actitud (Chinellato, 2015). Esta consideración comprende una triple base teórica: componentes cognitivos, afectivos y conductuales. Estos tres componentes se retroalimentan entre sí y constituyen la base para la consideración de la realidad por parte del individuo, así como de una colectividad. En este punto, los esquemas de representación aludidos configuran los factores tanto mentales como socio-históricos que influyen en la gestión de la información por parte tanto de un sujeto como de una comunidad cultural. La lengua, como herramienta fundamental para la comunicación, se nutre de estas configuraciones basadas en la triple organización componencial aludida anteriormente. Se trata, por tanto, de un sistema de comunicación eminentemente cognitivo y mentalista (Chomsky, 1957; 1965), el cual también tiene en cuenta los factores sociohistóricos, sociales y culturales vinculados a las distintas comunidades de habla insertas en una misma comunidad lingüística (Halliday, 1979).

Las actitudes lingüísticas, por tanto, consideran el conocimiento y la experiencia personal. Esta dualidad las vincula con el componente cognitivo, al tiempo que también 
tiene presente los sentimientos derivados de la experiencia, lo cual se vincula con el componente afectivo. Por último, la inclinación a actuar de una u otra manera fruto tanto del conocimiento como de la experiencia se asocia con el componente conductual. Estos tres componentes son los que nos permiten distinguir entre las actitudes lingüísticas frente a las reacciones lingüísticas. Las primeras presentan unos patrones de durabilidad a pesar de albergar factores con una base estático-dinámica. Las segundas responden a un patrón de respuesta mucho más inmediato y espontáneo (Quesada, 2019).

\section{Actitudes lingüísticas hacia el aprendizaje/adquisición de una lengua extranjera/L2}

La lengua, como sistema de signos, se estructura en diferentes niveles o planos desde un punto de vista descriptivo (Coseriu, 1986). De este modo, las actitudes lingüísticas pueden ser susceptibles de concretarse en uno 0 varios planos lingüísticocomunicativos. Esto se ha visto potenciado en la actualidad por el enfoque comunicativo a la hora de aprender/adquirir una LE/L2. Así pues, si aplicamos un enfoque basado en las finalidades discursivas, el enfoque comunicativo actual comprende de una manera general cuatro destrezas básicas a partir de las cuales se superponen los diferentes niveles o planos lingüísticos aludidos supra: comprensión y expresión orales frente a comprensión y expresión escritas.

Esta base teórica ha sido uno de los pilares para la configuración de las actuales destrezas lingüísticas recogidas en el Marco Común Europeo de Referencia (MCER). Actualmente, dicho documento considera como principales destrezas o habilidades comunicativas la expresión oral, la expresión escrita, la comprensión auditiva, la comprensión lectora y la interacción (Consejo de Europa, 2001).

Estas destrezas se relacionan con el componente cognitivo de la lengua, a partir del conocimiento lingüístico-comunicativo que adquiera el alumno. Este conocimiento constituye uno de los pilares componenciales de la actitud hacia esa LE/L2. En este punto, el conocimiento lingüístico-gramatical tanto de una L1 como de una LE/L2 correlaciona directamente con la valoración de la misma por parte del hablante en el primer caso y del alumno en el segundo. De esta manera, un mayor conocimiento gramatical implica una mejor actitud del sujeto hacia dicha lengua o modalidad lingüística. Esto demuestra la relación existente entre el componente cognitivo y el componente afectivo en lo que respecta a las actitudes lingüísticas (Minera, 2009).

Esta correlación entre el componente cognitivo y el afectivo ha motivado en la presente investigación la consideración del conocimiento lingüístico-gramatical, así como de otros planos, a la hora de diseñar un cuestionario de valoración de actitudes hacia una herramienta de aprendizaje/adquisición de lenguas. Para ello hemos tenido en cuenta la formulación de ítems en relación con las destrezas lingüístico-comunicativas aludidas.

Por otro lado, fruto de la superación de los procesos de aprendizaje/adquisición de lenguas a partir de un enfoque descriptivo de los niveles o planos lingüísticocomunicativos, tuvimos también en cuenta la valoración por parte de los sujetos del enfoque por tareas llevado a cabo a través de la herramienta. Así pues, el diseño de la app PAPUA ha tomado en consideración tanto las destrezas aludidas como la organización de tareas para la enseñanza de una LE/L2 (Nunan, 2004), lo que también se ha tenido en cuenta en la formulación y selección de ítems del cuestionario elaborado. 
Asimismo, más allá de los conocimientos lingüístico-comunicativos y la organización de tareas y su relación con el componente afectivo, la cuestión de las creencias y actitudes hacia el aprendizaje/adquisición de una LE/L2 ha sido ampliamente estudiada tanto desde una perspectiva constructivista (Martínez, 2005) como cognitivista (Clément \& Kruidenier, 1983). Más recientemente, como señala Minera (2009), han surgido numerosos estudios acerca del análisis y cuantificación de las actitudes de los alumnos hacia el aprendizaje/adquisición de una LE/L2, especialmente acerca del componente afectivo y las posibles variables que contribuyen a la cuantificación del mismo (Clément, Dörnyei \& Noel, 1994). Así pues, además de los dos criterios aludidos para la formulación de ítems del cuestionario, tuvimos en cuenta el componente conductual como constructo perteneciente a la tripartición de los componentes de la actitud para la formulación y agrupamiento de los ítems, en virtud de la relación de interdependencia que existe entre el componente cognitivo, afectivo y conductual, así como a la relación que existe entre actitudes y competencias. Estas influyen en la ejecución de la actitud de manera directa (Azjen, 2001; Ajzen \& Fishbein, 2005).

\section{Actitudes hacia las herramientas digitales en el aprendizaje/adquisición de una LE/L2}

Uno de los itinerarios de investigación en los que ha derivado el uso de las nuevas tecnologías en los procesos de aprendizaje/adquisición de una LE/L2 es en la evaluación por parte tanto de docentes como de alumnos de dichas herramientas digitales. Esto ha dado lugar al estudio de las creencias y actitudes de dichos agentes acerca de las Tecnologías de la Información y Comunicación (en adelante TIC) en el aula, especialmente en los periodos iniciales de formación del profesorado (Tapia, 2018). Todo ello ha suscitado un enorme interés acerca de las actitudes por parte de estudiantes y profesorado sobre el uso de las TIC en los procesos de enseñanzaaprendizaje (Albirini, 2006; Drossel, Eickelmann, \& Gerick, 2017; Escorcia-Oyola \& Jaimes de Triviño, 2015).

Este tipo de investigaciones ha dado lugar, básicamente, a tres tipos de estudio fundamentales:

- El estudio cuantitativo, llevado a cabo a partir de la recogida de datos mediante cuestionarios diseñados para tal efecto. Este tipo de investigaciones suele dar lugar a estudios de tipo descriptivo-correlacional, mediante los cuales se cruzan variables dependientes e independientes entre sí a la vista de averiguar patrones de conducta según los diversos componentes del concepto actitud (Sáez, 2010; Simón, 2013).

- El estudio cualitativo, llevado a cabo generalmente mediante cuestionarios y/o entrevistas dirigidas y semidirigidas. A partir de la información recogida, el investigador suele hacer una interpretación de las respuestas. En la actualidad existen diversos enfoques como la denominada Teoría Fundamentada (Glasser \& Strauss, 1967; Strauss, 1988).

- La combinación de los enfoques inmediatamente anteriores a partir de la triangulación de datos mediante estadística descriptiva y contenido extraído de documentos semiformales (Cardona, Fandiño \& Galindo, 2014).

En la actualidad, se distinguen básicamente dos posturas complementarias para el constructo actitud hacia las TIC: las TIC como objeto de actitud y, por otro lado, la integración didáctica de las TIC. Para ello, tres factores fundamentales para la construcción de la actitud hacia las TIC son los conocimientos y habilidades TIC, la disciplina formativa en la que se especializan los profesores y las creencias sobre el proceso de aprendizaje y su relación con las TIC (Tapia, 2018). 
En este punto, de acuerdo a la presente investigación, consideramos fundamentales dos componentes: la actitud hacia las TIC por parte de los informantes que compusieron la muestra, esto es, futuros docentes de Educación Primaria y Secundaria y, por otra parte, la actitud hacia el aprendizaje/adquisición de una LE/L2. Ambos constructos constituyeron las dimensiones para el diseño de nuestra investigación.

\section{Diseño de la investigación}

Para diseñar el cuestionario cuantitativo, con preguntas cerradas escala Likert, tuvimos en cuenta primeramente las variables que habíamos aislado a través del estudio analítico de dos documentos internos aportados por la empresa que ha generado la herramienta PAPUA, Aprendizaje Inmersivo S.L., así como a través de la realización de entrevistas cualitativas a dos informantes claves del equipo que había creado la herramienta tecnológica. Asimismo, optamos por la propia experimentación con la app con rol de usuario y la web con rol de profesor por parte de los investigadores para conocer y profundizar en esta herramienta tecnológica.

Esta aplicación, PAPUA, comprende unos contenidos lingüísticos adaptables entre el nivel $A 1$ y el nivel $C 1$ según el MCER. Asimismo, propone el trabajo de las diferentes destrezas lingüístico-comunicativas (leer, escribir, hablar, escuchar e interactuar) mediante actividades insertas en situaciones comunicativas y contextos basados en la vida diaria. Se propone, por tanto, un aprendizaje/adquisición integral de una LE/L2 a partir de los siguientes componentes y tareas: social knowledge skills, casual writings, inmersive readings, pronuntiation accuracy, interactive speaking and visual listening. Se trata, consecuentemente, de un aprendizaje integrado por tareas (integrated tasks) de acuerdo a la interacción social del aula (Feryok, 2017; Gómez \& Rodríguez, 2008; Morton, 2016). El destinatario de dicha herramienta lo constituyen alumnos a partir de 13 años en adelante. Se trata de un programa descargable según una aplicación para móvil y tableta.

En una segunda fase, realizamos un análisis de la literatura científica sobre el aprendizaje de lengua LE/L2 para enmarcar la investigación encontrando un paradigma para medir las actitudes y creencias acerca de la herramienta por parte de la muestra investigada. A partir de este marco, elaboramos un cuestionario de 23 ítems. Dicho cuestionario se basó en una escala Likert de cinco posibilidades de respuesta en gradación ascendente: poco, algo, bien, bastante, mucho. Para su diseño, en primer lugar, seleccionamos un total de 23 ítems redactados en lengua española e inglés. Dichos ítems se agruparon en torno a dos variables fundamentales: eficacia de la herramienta para el aprendizaje/adquisición de las destrezas orales de una LE/L2; eficacia de la herramienta para el aprendizaje/adquisición de las destrezas escritas de una LE/L2. Paralelamente, seleccionamos ítems que tuvieran en cuenta los tres componentes de la actitud (cognitivo, afectivo y conductual) hacia la herramienta y su eficacia con respecto al aprendizaje/adquisición de las destrezas orales y escritas reseñadas en el marco teórico según un enfoque integrado de tareas (integrated learning by integrated tasks). Esto nos llevó a tener en cuenta la variable interactividad, la cual se encuentra refrendada por la metodología comunicativa contextualizada del aprendizaje/adquisición de una LE/L2 (Mayor, 1994). Por último, tuvimos también en cuenta el ámbito de la motivación del alumno en relación con la herramienta y sus posibilidades de eficacia, es decir, intentamos cuantificar el grado de motivación de aprendizaje/adquisición del alumno en relación con el aprendizaje/adquisición de una LE/L2 en relación con la herramienta (Minera, 2010).

Este cuestionario bilingüe de 23 ítems elaborado ad hoc, una vez lo terminamos, fue depurado y validado por un panel de expertos compuesto por cuatro expertos del campo 
educativo universitario: dos metodólogos, un filólogo y un pedagogo. La validación de expertos se sostiene en el campo educativo por Escobar y Cuervo (2008) y en lingüística aplicada por Robles \& Rojas (2015). Así pues, a estos expertos se les contextualizó en la indagación y se les presentó el cuestionario inicial de 23 ítems. Estos expertos estudiaron esta herramienta en referencia a claridad, adecuación y pertinencia. Emitieron sugerencias que se incorporaron para componer la versión definitiva de 23 preguntas. Por tanto, los expertos colaboraron con la redacción y depuración de dichos ítems hasta que el cuestionario quedó perfectamente organizado en torno a las dimensiones mencionadas supra: Actitudes lingüísticas hacia el aprendizaje/adquisición de una lengua extranjera/L2 y Actitudes hacia las herramientas digitales en el aprendizaje/adquisición de una LE/L2.

Para el estudio de ambas dimensiones tuvimos en cuenta una serie de variables en el cuestionario de exploración de 23 ítems que exponemos a continuación (tabla 1). Estas variables se organizaron en torno a los componentes adyacentes a la actitud lingüística hacia el aprendizaje/adquisición de una LE/L2 y actitudes hacia las herramientas digitales en el aprendizaje/adquisición de una LE/L2. Estos componentes fueron la actitud según la eficacia lingüístico-comunicativa, la actitud según la eficacia de interactividad y la actitud según la eficacia motivacional. Para ello tuvimos en cuenta tanto los componentes del concepto actitud (cognitivos, afectivos y conductuales) como los contenidos y competencias del MCER.

Tabla 1. Variables que componen el cuestionario de exploración

Actitud según la eficacia lingüístico-comunicativa

1. ¿Crees que las actividades de la app de Papua facilitan la soltura en la comunicación informal porque favorece la capacidad de respuesta en una situación de interacción social, real, contextualizada?

2. ¿Crees que las actividades de la app de Papua de inteligencia artificial, que reconoce cómo pronuncias, mejora la pronunciación de la LE/L2?

3. ¿Crees que las actividades de la app de Papua mejora la comprensión lectora de una LE/L2?

4. ¿Crees que las actividades de la app de Papua respecto a la lectura mejora la memorización de palabras?

¿Crees que las actividades de la app de Papua mejora la comprensión escrita de una LE/L2?

¿Crees que las actividades de la app de Papua mejoran la redacción correcta de una LE/L2?

¿Crees que las actividades de la app de Papua facilitan la mejora del vocabulario y frases hechas de una LE/L2?

¿Crees que las actividades de la app de Papua mejora (refuerza) el conocimiento morfosintáctico de una LE/L2?

9. ¿Crees que la interpretación emocional de los actores en una situación real a través de las actividades de la app de Papua mejora la memorización de una LE/L2?

10. ¿Crees que las actividades con repetición de contenidos mejoran la retención memorística?

Actitud según la eficacia de la interactividad

11. ¿Crees que las actividades de la app de Papua facilitan la comprensión oral en la comunicación informal porque favorece la capacidad de respuesta en una situación de interacción social, real, contextualizada?

12. ¿Crees que las actividades de la app de Papua facilitan que uno mejore en saber comportarse con los demás y en un contexto diferente al propio, en este caso norteamericano?

13. ¿Crees que la interpretación emocional de los actores en una situación real a través de las actividades de la app de Papua me jora la morfosintaxis de una LE/L2?

14. ¿Crees que la interpretación emocional de los actores en una situación real a través de las actividades de la app de Papua mejora el vocabulario de una LE/L2?

15. ¿Crees que poder responder en el papel de uno mismo con las emociones que a uno le afectan, mejora la memorización de una LE/L2?

16. ¿Crees que poder responder en el papel de uno mismo con las emociones que a uno le afectan, mejora la morfosintaxis de una LE/L2?

17. ¿Crees que poder responder en el papel de uno mismo con las emociones que a uno le afectan, mejora el vocabulario de una LE/L2?

Actitud según la eficacia motivacional

18. ¿Crees que las actividades de la app de Papua facilitan la confianza en uno mismo de poder hablar inglés en la comunicación informal porque favorece la capacidad de respuesta en una situación de interacción social, real, contextualizada?

19. ¿Crees que la autoevaluación de varios aspectos (promedio de pronunciación y de respuesta) de las actividades en cada escena de la app de Papua mejora la motivación intrínseca a favor de aprender/adquirir una LE/L2?

20. ¿Crees que la autoevaluación de varios aspectos (pronunciación correcta) de las actividades en cada escena de la app de Papua mejora la motivación intrínseca a favor de aprender/adquirir una LE/L2?

21. ¿Crees que la autoevaluación de varios aspectos (respuestas de contenido erróneas) de las actividades en cada escena de la app de Papua mejora la motivación intrínseca a favor de aprender/adquirir una LE/L2?

22. ¿Crees que la autoevaluación de varios aspectos (errores gramaticales) de las actividades en cada escena de la app de Papua mejora la motivación intrínseca a favor de aprender/adquirir una LE/L2?

23. ¿Crees que la autoevaluación de varios aspectos (errores sociales) de las actividades en cada escena de la app de Papua mejora la motivación intrínseca a favor de aprender/adquirir una LE/L2?

En una tercera fase, para validar este cuestionario inicial de 23 ítems y conseguir el cuestionario validado, la presente investigación tomó como muestra a 31 alumnos 
matriculados en el tercer curso del Grado de Educación Primaria de la Universidad de Huelva durante el curso 2020/2021. Se trata, por tanto, de un muestreo por conveniencia, puesto que nuestra intención fue considerar informantes que cumplieran con las características del estudio. En este caso, estudiantes del tercer curso del Grado en Educación Primaria. Es un muestreo no probabilístico, puesto que no todos los estudiantes del Grado en Educación Primaria tuvieron la oportunidad igual e independiente de participar en el muestreo. Exclusivamente los del tercer curso. Estos informantes participaron en la investigación de manera voluntaria, habiendo sido informados previamente acerca del propósito de la investigación. La edad media fue de 23.2 (d.t.). El $71 \%$ de los informantes fueron chicas. El $77.8 \%$ de los informantes posee el nivel B1 de inglés LE/L2.

A partir de ahí comprobamos el índice de fiabilidad de este primer cuestionario. El total de 23 ítems dio el siguiente resultado para cada uno de dichos ítems de la escala. El Alpha de Cronbach inicial fue de .81 .

Si bien la fiabilidad inicial del cuestionario inicial fue alta, observamos un cierto desnivel en la ponderación de los diferentes ítems. Asimismo, pensamos que el número de ítems de la escala podía resultar excesivo a la hora de aplicar el cuestionario a los informantes. Por estos motivos decidimos reelaborar el cuestionario para quedarnos con un número menor de ítems. Esto nos llevó a establecer unas categorías definitivas a la hora de agrupar dichos ítems y a reducir su número a 13 ítems. Nuevamente, el cuestionario fue sometido a la evaluación de expertos para su depuración y para la redacción definitiva de los ítems.

A partir de ahí, llevamos a cabo un análisis factorial tanto exploratorio como confirmatorio para establecer los factores de medición estadística, con el fin de comprobar si dichos factores se corresponden con los constructos de medición propuestos inicialmente. Estos constructos motivaron la evaluación de los expertos para la redacción y selección de ítems. Los resultados fueron los siguientes.

\section{Resultados}

Como comentamos, tras la depuración del cuestionario bilingüe en lengua española e inglés, la escala contó con un total de 13 ítems. El alfa de Cronbach fue de .79. Asimismo, mostramos los estadísticos de escala (tabla 2).

\begin{tabular}{|lccc|}
\hline \multicolumn{3}{c|}{ Tabla 2. Estadísticos de la escala } \\
\hline Media & Varianza & Desviación típica & N elementos \\
54,4194 & 46,918 & 6,84969 & 13 \\
\hline
\end{tabular}

El nivel de fiabilidad de la escala final resultó válido, así como los estadísticos de la misma. Para la validación del instrumento utilizamos el programa estadístico SPSS versión 21.

Los 23 ítems iniciales comprendieron más preguntas específicas acerca de la actitud hacia la herramienta según su eficacia con respecto a las destrezas comunicativas y sus componentes internos. No obstante, consideramos más relevante ponderar los ítems que, más allá de la eficacia lingüístico-comunicativa de la misma con respecto a las destrezas, ponderaron aspectos como la interactividad y la motivación. La razón principal es que estas dos variables constituyen la base de la originalidad de la presente app y los factores que organizaron el cuestionario de acuerdo a las dos dimensiones de medición originales. 
Para comprobar estas cargas factoriales llevamos a cabo la prueba de esfericidad de Kaiser-Meyer- Olkin y prueba de Barlett. Asimismo, utilizamos el método de rotación Varimax. Los resultados mostraron un valor de .48 para la prueba KMO (tabla 3). Se trata de un valor bajo, por lo que decidimos nuevamente depurar el cuestionario y eliminar los ítems que mostraron mayor desviación típica (ítems 7 y 13).

\begin{tabular}{|cccc|}
\hline \multicolumn{4}{|c|}{ Tabla3. Prueba KMO } \\
\hline Media Kaiser-Meyer-Olkin & Chi-cuadrado aproximado & Esfericidad de Barlett & Sig. \\
.48 & 155,486 & 105 & .001 \\
\hline
\end{tabular}

Tras la nueva depuración del cuestionario, el índice KMO fue de 5.1 (tabla 4) y el Alpha de Cronbach fue de .73. Si bien es cierto que el valor de la prueba KMO es algo bajo, sí confirma los dos factores en los que dividimos estadísticamente los ítems de acuerdo a las dos dimensiones propuestas, ya que el nivel de significatividad es de 0.01 . El valor del Alpha de Cronbach final, aunque disminuyó con respecto al valor original, resultó válido. Para la escala final véase el Anexo de la presente publicación.

\begin{tabular}{|cccc|}
\hline \multicolumn{4}{|c|}{ Tabla 4. Prueba KMO } \\
\hline Media Kaiser-Meyer-Olkin & Chi-cuadrado aproximado & Esfericidad de Barlett & Sig. \\
.51 & 125,457 & 78 & .001 \\
\hline
\end{tabular}

\section{Discusión}

Como hemos comprobado, el cuestionario reúne las condiciones necesarias tanto psicométricas como teóricas. Así pues, el Cuestionario para la medición de actitudes de aprendizaje y actitudes lingüísticas de una LE/L2 resulta fiable y válido. La muestra de estudiantes a partir de los cuales se llevó a cabo la validación del instrumento $(\mathrm{N}=31)$ ratificó la utilidad del mismo a la hora de evaluar las dos dimensiones establecidas.

El presente instrumento constituye un complemento para otras escalas relacionadas con la eficacia de las apps en relación con el ámbito lingüístico-comunicativo (Del Moral et al, 2018; Pinto, M.; Gómez-Hernández, J.A.; Sales, D. et al., 2019) o webs (Gómez et al, 2019). La principal novedad que aporta nuestro instrumento es, por un lado, que se trata de un proceso de aprendizaje/adquisición de una LE/ L2 y, por otro, que se trata de una app programada desde el ámbito de la inteligencia artificial. Esto dota al alumno a la hora de gestionar la información en los diferentes escenarios de aprendizaje de una mayor flexibilidad y creatividad, lo que redunda especialmente en las dimensiones interactividad y motivación.

En relación a otros campos de estudio, se han diseñado dos instrumentos de evaluación de apps Ilamado "Mobile App" para el campo de Ciencias Sociales en estudios superiores dirigido tanto para profesorado como alumnado por parte de Pinto et al (2019). Este instrumento no se encuentra plasmado en la publicación. También se ha realizado un estudio evaluativo de una app de Ciencias Sociales, Architecture gothique/romane, por parte de Kortabiarte et al (2018). No se aporta el cuestionario en la publicación. Se pregunta por la satisfacción de uso de la app, percepción de aprendizaje y utilidad en el aula. Con respecto al aprendizaje, el alumnado reconoce adquirir conocimientos técnicos y teóricos de la arquitectura. Los ítems de aprendizaje en este cuestionario "CAAAL" han reflejado más aspectos que este otro estudio de la app de arquitectura.

Con todo, CAAAL presenta algunas carencias en comparación con estos cuestionarios. La principal es que no se han investigado las dimensiones de satisfacción y utilidad en el aula. Esto nos lleva a plantear de manera prospectiva la posibilidad de crear un instrumento complementario al presente o bien de sugerir la complementación en la 
investigación para estas dimensiones a partir de otros instrumentos de tipo cualitativo. Tal es el caso, por ejemplo, de la entrevista semiestructurada.

En cualquier caso, en la actualidad hemos encontrado referencias confrontadas con respecto al uso de apps para el aprendizaje/ adquisición de una LE/L2. Así pues, Álvarez (2020) alaba apps como Duolingo o Babbel para comenzar a aprender/adquirir una LE/L2, por ganar en soltura y por la ludificación. En cambio, este académico pone en duda la eficacia de las apps de aprendizaje/adquisición de una LE/L2 para especializarse en un campo profesional, ya que las considera descontextualizadas y basadas en la repetición de la traducción.

Por otra parte, Jiménez \& Martínez (2019) realizan una clasificación dual de apps con las que se crea álbum ilustrado mediante storytelling. Primero, apps con imágenes artísticas como Storybird. Segundo, apps en las que se puede incluir audio o vídeo como Scratch.

En relación a otros campos, Arceo-Vacas et al (2019) dispusieron de una app para que los alumnos universitarios evaluaran la calidad del profesorado.

El instrumento expuesto en la presente investigación posee la ventaja de abordar dimensiones tanto intralingüísticas como extralingüísticas. En este punto, pensamos que resulta un cuestionario más completo que los instrumentos examinados hasta ahora. Como principal carencia, sin embargo, consideramos que puede resultar demasiado ambicioso de acuerdo a la diversidad y al número de dimensiones que aborda (cuatro en total) en un esfuerzo de síntesis de ítems, de modo que el instrumento final cuenta con trece preguntas.

Asimismo, la génesis y validación del cuestionario se ha realizado a partir de una app, con las cualidades limitadas de esta app para el aprendizaje/adquisición de una LE/L2. De este modo, si bien todas las destrezas comunicativas descritas por el MCER son trabajadas, es cierto que hay una preponderancia de las destrezas orales por encima de las escritas. Una de las posibles limitaciones del cuestionario sea la mayor o menor adaptabilidad del mismo a otras apps (donde adquieran mayor protagonismo las destrezas escritas) o que presenten otras funciones, cualidades o fines.

En cualquier caso, la primera fortaleza de este cuestionario CAAAL consiste en proponer ítems a partir de una app en la que se ha contrastado la mejora en el aprendizaje/adquisición de una LE/L2. Se trata, por tanto, de una app particular que incluye un tipo de tecnología de programación avanzada en una de sus funciones, la de reconocimiento de voz del usuario. Por otro lado, la cualidad de este cuestionario es que se pueda aplicar al estudio evaluativo de otras apps de aprendizaje/adquisición de una LE/L2 para evaluar el proceso de enseñanza-aprendizaje del alumnado en todo este espectro de aspectos, con contextualización de los escenarios de aprendizaje adaptable a diferentes niveles de competencia mediante la diversificación de ejercicios y su dificultad.

Como conclusión pensamos que el cuestionario CAAAL, creado a partir de los resultados positivos acerca de actitudes lingüísticas y aprendizaje de la lengua que se han extraído la app PAPUA, puede ser un instrumento pionero en la medición de las dimensiones actitud de aprendizaje y actitudes lingüísticas de una app de aprendizaje/adquisición de una lengua extranjera/L2. Las principales ventajas que aporta son la medición de dimensiones tanto intralingüísticas como extralingüísticas, así como la dimensión motivacional del proceso. Esta diversidad de dimensiones permite la 


\section{adaptabilidad de la prueba a otras apps conforme este tipo de herramientas de aprendizaje se generalicen.}

\section{Referencias} ALBIRINI, A. “Teachers' attitudes toward information and communication technologies: the case of Syrian
EFL teachers". Computers \& Education, 47(4), 373-398 (2006).

ÁLVAREZ, F.J. "Hasta qué punto son fiables las apps para aprender idiomas. The conversation", 1-3 (2020).

AJZEN, I \& FISHBEIN, M. "The Influence of Attitudes on Behavior". En D. Abarracin, D., Johnson, B.T. \& Zanna, M.P. (Eds.). "The Handbook of Attitudes", 173-222. Mahwah, NJ: Lawrence Enrbaum Associates, Inc

ARCEO-VACAS, A., NIÑO-GONZÁLEZ, JII. \& ÁLVAREZ-SÁNCHEZ, S. "Uso de una app móvil para evaluar la calidad de la enseñanza superior". Prisma social: revista de investigación social, 27, $65-85$ (2019).

APRENDIZAJE INMERSIVO S. L. Papuation.com. "Saca el inglés que llevas dentro" (s.f.).

BANDURA, A. "Social Leaming Theory". Englewood Cliffs, New Jersey: Prentice-Hall (1976).

BRUNER, J. "Acción, pensamiento y lenguaje". Madrid: Alianza (1984).

BRUNER, J. "El habla del niño". Buenos Aires: Paidós (1983).

CARDONA, A., FANDIÑO, Y. J. \& GALINDO, J.A. "Formación docente: creencias, actitudes y competencias para el uso de TIC". Lenguaje, 42(1), 175-208 (2014).

CLÉMENT, R; DÖRNYEI, Z. \& NOELS. K. A. "Motivation, self-confidence and group cohesion in the foreign language". Language Learning, 44 (3), $417-448$ (1994).

CONSEJO DE EUROPA. "Marco común europeo de referencia para las lenguas: aprendizzaje, enseñanza, evaluación", (2002). Madrid: Ministerio de Educación, Cultura y Deporte (2001).

COPE, B. \& KALANTZIS, M. Mutiliteracies: "New Literacies, New Leaming". Pedagogies: An International Joumal, 4(3), $164-195$ (2009)

COSERIU, E. "Lecciones de lingüistica general". Madrid: Gredos (1986). CROSS, T. "Mother's speech adjustment: the contributions of selected child listener variables". En Snow,
C.E. \& Ferguson, C.H.A. "Talking to children: Language input and acquisition". London: Cambridge University C.E. \& Fergus
Press (1977).

CHINELLATO, A. "Actitudes lingüisticas en la frontera Venezuela-Brasil. Tesis de Maestria". Universidad de los Andes, Facultad de Humanidades y Educación (2015).

CHOMSKY, A.N. "Syntactic structures". The Hague/Paris: Mouton \& Co (1957).

CHOMSKY, A.N. "Aspects of the Theory of Syntax". Cambridge, Massachusetts: MIT Press (1965)

DEL MORAL PÉREZ, M.E., BELLVER, M.C. \& GUZMÁN, A.P CREAPP K6-12: "Instrumento para evaluar la potencialidad creativa de app orientadas al diseño de relatos digitales personales". Digital Education
Review, 33, 284-305 (2018).

DIAZ, C.H., \& JANSSON, L. "El aprendizaje del inglés y el uso de tecnologias: percepciones de estudiantes y profesores de inglés del nivel secundario chileno". Revista electrónica Matices en lenguas extranjeras, 5,

ESCOBAR-PÉREZ J. \& CUERVO-MARTINEZZ, A. "Validez de contenido y juicio de expertos: una aproximación a su utilización". Avances en medición, 6, $27-36$ (2008).

DROSSEL, K; EICKELMANN, B. \& GERICK, J. "Predictors of teachers' use of ICT in school - the relevance of school characteristics, teachers' attitudes and teacher collaboration". Education and Information Technologies, 22(2), 551-573 (2017). EAGLY, A.H. \& CHAIKEN, S. "Attitude strength, attitude structure, and resistance to change". En R.E., Petty, Enbaum, $413-432$ (1995)

EAGLY, A.H. \& CHAIKEN, S. "Attitude structure and function". En D.T., Gilbert, S.T., Fiske \& G., Lindzey (Eds.) "The Handbook of Social Psychology, Vol 1" "New York: MoGriw Hill, 269322 , thed (1998)

ECHEVERRIA M. "Aplicaciones computacionales para la enseñanza aprendizaje del español como lengua matema". Revista de Estudios de Adquisición de la Lengua Española REALE, 1, 25-50 (1994).

ESCORCIA-OYOLA, L. \& JAIMES DE TRIVIÑO, C. "Tendencias de uso de las TIC en el contexto escolar a partir de las experiencias de los docentes". Educación y Educadores, 18(1), 137-152 (2015).

FERYOK, A. "Sociocultural Theory and Task- Based Language Teaching: The Role of Praxis". TESOL Quarterly, 51(3), $716-727$ (2017). GARCIAA-ARETIO, L. "El juego y otros principios pedagógicos. Supervivencia en la educación a distancia y
virtual". RIED: Revista lberoamericana de Educación a Distancia. AIESAD (19). 1, 9-23 (2016).

GARCIAA-VALCÁRCEL MUÑOZ-REPISO, A. \& MARTiN DEL POZO, M. "Análisis de las competencias digitales de los graduados en titulaciones de maestro". Revista latinoamericana de tecnologia educativa, 15(2), 156-168 (2016)

GLASER B. \& STRAUSS, A. "The discovery of Grounded theory: Strategies for qualitative research". New York: Aldine Publishing (1967).

GÓMEZ PARRA, M.E;; HUERTAS ABRIL A. C. \& ESPEJO MOHEDANO, R. "Estudio para la validación de dos instrumentos para la evaluación de dos stitos web de aprendizaje de lenguas extranjeras". Revista de lenguas para fines especificos, 25(1), $62-80$ (2019).

GÓMEZ, S., \& RODRIGUEZ, E. "The use of Integrated-Tasks in speaking". Cuenca: Universidad de Cuenca (2008).

GONZÁLEZ, K.J. " "Influencia de los recursos digitales en la enseñanza y el aprendizaje del idioma inglés en las escuelas normales de Oaxaca, México". Revista Boletin Redipe, 9(7), 150-164 (2020).

HALLIDAY, M.A.K. "El lenguaje como semiótica social". México: Fondo de Cultura Económica (1979).

IZQUIERDO S. "Actitudes ante el deterioro de la lengua. Español en Brasil". Marco ELE. Revista didáctica de español como lengua extranjera, 13. Julio-diciembre de 2011 (2011).

JIMÉNEZ-PÉREZ, E. \& MARTINEZZ-LEÓN, N. "Innovación educativa para futuros maestros: enseñar el uso de herramientas TIC para literatura infantil y juvenil (LIJ)". En Gázquez Linares, J.J. et al (Coords.). "Innovación docente e investigación en arte y humanidades". Madrid: Dykinson (2020).

KORTABITARTE, A., GILLATE, I., LUNA, U. \& IBÁÑEZ- ETXEBERRIA, A. "Las aplicaciones móviles como recursos de apoyo en el aula de Ciencias Sociales: estudio exploratorio con la app 'Architecture 33(1), $65-79$ (2018). LU, M.M. "Effectiveness of vocabulary leaming via mobile phone". Journal of Computer Assisted Leaming,
(24). 6. $515-525(2008)$.

MARTíN, M.C. "Diseño y validación de cuestionarios". Matronas profesión, 5(17), 23-29 (2004).

MARTINNEZ, J. "Creencias relativas al aprendizaje de una lengua extranjera". Cauce, revista internacional MARTINEZ, J. "Creencias relativas al aprend
de filologia y su didáctica, 28, 219-234 (2005). MAYOR, J. "La adquisición de una segunda lengua". En Sánchez-Lobato, J. y Santos-Gallego, I. (coords.).
"Problemas y métodos de la enseñanza del español como lengua extranjera", 21-60. Madrid: Sgel (1994).

MAYOR, J. "La actividad lingüistica entre la comunicación y la cognición". En Mayor, J. \& Pinillos, J.L. (dirs.). "Comunicación y lenguaje". Madrid: Alhambra (1991).

MINERA, LE. "El cuestionario MAALE, técnica para recolección de datos de las variables afectivas, motivación y actitudes en el aprendizaje de una lengua extranjera". RedELE: Revista Electrónica de
Didáctica ELE, (19) (2010).

MINERA, LE. "El papel de la motivación y las actitudes en el aprendizaje de ELE en un contexto de enseñanza formal para adultos alemanes". Revista Nebrija de Lingǘstica Aplicada, $6,58-73$ (2009).

MORTON, T. "Conceptualizing and investigating teachers' knowledge for integrating content and language (2016).

NUNAN, D. "Task-Based Language Teaching". Cambridge: Cambridge University Press (2004).

PARALES-QUENZA, C.J. \& VIZCAINO-GUTIÉRREZ, M. "Las relaciones entre actitudes y relaciones sociales". Revista latinoamericana de Psicologia, 39(2), 351-361 (2006).

PINTO, M.; GÓMEZ-HERNÁNDEZ, J.A.; SALES, D. ET AL. "Learning and teaching digital skills in a mobile environment: advances of a research on university professors and students of Social Sciences degree". RICl. Revista Ibero-Americana de Ciência da Informaçâa, 12(2), 558-596 (2019).

PRENSKY, M. "Digital Natives, Digital Immigrants". On the horizon, 9(5), 1-6 (2001).

QUESADA, M.A. "Actitudes lingüisticas de los hispanohablantes hacia su propia lengua: nuevos alcances". Zeitschrift für Romanische Philologie, 135(1), 158-194 (2019). ROBLES GARROTE, P. \& ROJAS, M. D. C. "La validación por juicio de expertos: dos investigaciones 
Cuestionario CAAAL -Cuestionario de actitudes de aprendizaje y actitudes lingüísticas hacia una L2-

El presente cuestionario mide las actitudes tanto lingüísticas como de aprendizaje de una lengua extranjera/L2 a través de una app. Es necesario que respondas todas las preguntas. Se trata de un cuestionario anónimo, de respuesta voluntaria e individual. Elige mediante un círculo la opción de respuesta que consideres más adecuada para cada una de las preguntas.

Género:

Edad:

Grado y curso que estudias:

Cualificación de idiomas:

1. ¿Crees que las actividades de la app facilitan la soltura en la comunicación informal porque favorece la capacidad de respuesta en una situación de interacción social, real, contextualizada? / Do you think that the activities of the app facilitate informal communication because it favours the ability to respond in a social, real and contextualized communication?

Poco Algo Bien Bastante Mucho

2. ¿Crees que las actividades de la app facilitan la comprensión oral en la comunicación informal porque favorecen la capacidad de respuesta en una situación de interacción social, real, contextualizada? / Do you think that the activities of the app facilitate oral comprehension in informal communication because they favor the ability to respond in a situation of social, real, contextualized interaction?

Poco Algo Bien Bastante Mucho

3. ¿Crees que las actividades de la app facilitan la confianza propia de poder hablar inglés en la comunicación informal porque favorece la capacidad de respuesta en una situación de interacción social, real, contextualizada? /Do you think that the activities of the app increase self-confidence to speak in English in informal communication situations because it favours the ability to respond in a situation of social, real, contextualized interaction?

Poco Algo Bien Bastante Mucho

4. ¿Crees que las actividades de la app mejoran la comprensión lectora de la lengua extranjera? / Do you think that the activities in the app improve a second language's reading comprehension?

Poco Algo Bien Bastante Mucho

5. ¿Crees que las actividades de la app respecto a la lectura mejoran la memorización de palabras? / Do you think that the activities of the app regarding reading skills improve the memorization of words?

Poco Algo Bien Bastante Mucho

6. ¿Crees que las actividades de la app mejoran el conocimiento morfosintáctico de la lengua extranjera? / Do you think that the activities of the app improve the morphosyntactic knowledge of the second language?

Poco Algo Bien Bastante Mucho

7. ¿Crees que la interpretación emocional de los actores en una situación real a través de las actividades de la app mejora el vocabulario de la lengua extranjera? I Do you think that actors' emotional interpretation in a real situation through the activities of the app improves the vocabulary of the second language?

Poco Algo Bien Bastante Mucho 
8. ¿Crees que poder responder en el papel propio con las emociones que a uno le afectan, mejora la memorización de la lengua extranjera? / Do you think that being able to answer as yourself with the emotions that affect you it improves the memorization of the second language?

Poco Algo Bien Bastante Mucho

9. ¿Crees que poder responder en el papel propio con las emociones que a uno le afectan, mejora el conocimiento de vocabulario en lengua extranjera? / Do you think that being able to respond in the role of yourself with the emotions that affect you improves your knowledge of vocabulary in the second language?

Poco Algo Bien Bastante Mucho

10. ¿Crees que las actividades con repetición de contenidos, o un enfoque en espiral, mejoran la retención memorística? / Do you think that activities with a spiral approach improve memory capability?

Poco Algo Bien Bastante Mucho

11. ¿Crees que la autoevaluación del promedio de pronunciación y de respuesta en cada escena de la app mejora la motivación intrínseca del usuario a favor de aprender la lengua extranjera? / Do you think that the self-assessment of the average pronunciation and response in each scene of the app improves the intrinsic motivation of the user in favour of second language learning?

Poco Algo Bien Bastante Mucho

12. ¿Crees que la autoevaluación de respuestas de contenido erróneas en cada escena de la app mejora la motivación intrínseca a favor de aprender la lengua extranjera? /Do you think that the self-evaluation of wrong content answers in each scene of the app improves the intrinsic motivation in favor of learning the second language?

Poco Algo Bien Bastante Mucho

13. ¿Crees que la autoevaluación de errores sociales en cada escena de la app mejora la motivación intrínseca a favor de aprender la lengua extranjera? / Do you think that the self-assessment of social scene in each scene of the app improves the intrinsic motivation in favour of second language learning?

Poco Algo Bien Bastante Mucho

@Peña-Acuña \& Crismán-Pérez, 2021. @Aprendizaje Inmersivo S.L. 2021 